\title{
RESEARCH
}

Open Access

\section{Longitudinal trajectories of Alzheimer's ATN biomarkers in elderly persons without dementia}

Meng-Shan $\operatorname{Tan}^{1+}{ }^{1}, \mathrm{Xi} \mathrm{Ji}^{2+}$, Jie-Qiong Li ${ }^{1}$, Wei Xu${ }^{1}$, Hui-Fu Wang ${ }^{1}$, Chen-Chen Tan ${ }^{1}$, Qiang Dong ${ }^{3}$, Chuan-Tao Zuo ${ }^{4}$, Lan Tan ${ }^{1,2}$, John Suckling ${ }^{5,6,7}$, Jin-Tai $Y_{u^{3 *}}$ (D) and Alzheimer's Disease Neuroimaging Initiative

\begin{abstract}
Background: Models of Alzheimer's disease (AD) pathophysiology posit that amyloidosis [A] precedes and accelerates tau pathology $[1]$ that leads to neurodegeneration [N]. Besides this A-T-N sequence, other biomarker sequences are possible. This current work investigates and compares the longitudinal trajectories of Alzheimer's ATN biomarker profiles in non-demented elderly adults from the Alzheimer's Disease Neuroimaging Initiative (ADNI) cohort.

Methods: Based on the ATN classification system, 262 individuals were identified before dementia diagnosis and accompanied by baseline and follow-up data of ATN biomarkers (CSF A 42 , p-tau, and FDG-PET). We recorded the conversion processes in ATN biomarkers during follow-up, then analyzed the possible longitudinal trajectories and estimated the conversion rate and temporal evolution of biomarker changes. To evaluate how biomarkers changed over time, we used linear mixed-effects models.

Results: During a 6-120-month follow-up period, there were four patterns of longitudinal changes in Alzheimer's ATN biomarker profiles, from all negative to positive through the course of the disease. The most common pattern is that A pathology biomarker first emerges. As well as the classical A-T-N sequence, other "A-first," "T-first," and "Nfirst" biomarker pathways were found. The N-A-T sequence had the fastest rate of pathological progression (mean 65.00 months), followed by A-T-N (mean 67.07 months), T-A-N (mean 68.85 months), and A-N-T sequences (mean 98.14 months).

Conclusions: Our current work presents a comprehensive analysis of longitudinal trajectories of Alzheimer's ATN biomarkers in non-demented elderly adults. Stratifying disease into subtypes depending on the temporal evolution of biomarkers will benefit the early recognition and treatment.
\end{abstract}

Keywords: Alzheimer's disease, ADNI, Amyloid, Biomarker, Tau, Neurodegeneration

\footnotetext{
* Correspondence: jintai_yu@fudan.edu.cn

${ }^{\dagger}$ Meng-Shan Tan and $\mathrm{Xi}$ Ji contributed equally to this work.

${ }^{3}$ Department of Neurology and Institute of Neurology, Huashan Hospital,

Shanghai Medical College, Fudan University, 12th Wulumuqi Zhong Road,

Shanghai 200040, China

Full list of author information is available at the end of the article
}

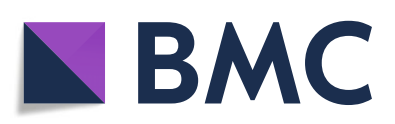

(- The Author(s). 2020 Open Access This article is licensed under a Creative Commons Attribution 4.0 International License, which permits use, sharing, adaptation, distribution and reproduction in any medium or format, as long as you give appropriate credit to the original author(s) and the source, provide a link to the Creative Commons licence, and indicate if changes were made. The images or other third party material in this article are included in the article's Creative Commons licence, unless indicated otherwise in a credit line to the material. If material is not included in the article's Creative Commons licence and your intended use is not permitted by statutory regulation or exceeds the permitted use, you will need to obtain permission directly from the copyright holder. To view a copy of this licence, visit http://creativecommons.org/licenses/by/4.0/. The Creative Commons Public Domain Dedication waiver (http://creativecommons.org/publicdomain/zero/1.0/) applies to the data made available in this article, unless otherwise stated in a credit line to the data. 


\section{Background}

Alzheimer's disease (AD) has a long preclinical phase that is characterized by accumulating pathology in the brain [1]. This pathology leads to detectable cognitive deficits in the preclinical stages of the disease [2]. The length of the preclinical phase has encouraged efforts to identify in vivo biomarkers to aid disease diagnosis and prognosis [3]. During this period, biomarkers derived from biofluids and brain imaging vary in continuous pathological processes that begin before the onset of symptoms $[1,4,5]$.

In 2018, the National Institute on Aging and Alzheimer's Association (NIA-AA) created a research framework to biologically define AD by ATN biomarkers (A $\beta$ deposition $[\mathrm{A}]$, pathologic tau $[\mathrm{T}]$, and neurodegeneration $[\mathrm{N}]$ ) and treat cognitive impairment as a symptom/ sign of the disease [6]. Models of AD pathophysiology theorize a temporal sequence in which amyloidosis [A] initiates a biological cascade, followed by pathologic tau aggregation $[\mathrm{T}]$ that leads to neurodegeneration $[\mathrm{N}][7$, 8]. Besides this classic A-T-N sequence, other biomarker sequences are possible [6]. For example, primary tauopathies can evolve towards $A \beta$ plaques before neurological signs are seen, that is, a T-A-N sequence. A current challenge in $\mathrm{AD}$ research is to identify the sequence of pathologic changes that occurs during the preclinical stages of disease by in vivo longitudinal study [9].

Based on this ATN classification system, our current work is investigating and comparing the longitudinal trajectories of Alzheimer's ATN biomarker profiles in non-demented elderly adults from the Alzheimer's Disease Neuroimaging Initiative (ADNI) using longitudinal follow-up data. The objective was to visualize which pathology biomarkers first emerge and how they change through the course of the disease. Understanding these longitudinal changes will provide insight into the pathophysiological progression of $\mathrm{AD}$ and potentially stratify the disease into subtypes depending on the temporal evolution of biomarkers.

\section{Methods}

\section{Study design}

The ADNI was launched in 2003 as a public-private partnership, led by principal investigator Michael W. Weiner, MD, VA Medical Center and University of California-San Francisco (http://www.loni.ucla.edu/ADNI). The ADNI was established to test whether serial MRI, PET, other biological markers, and clinical and neuropsychological assessment can be combined to measure the progression of mild cognitive impairment (MCI) and early AD. For more information, see http://www.adni-info.org.

\section{Participants}

All participants included in this study were enrolled in the ADNI database (http://adni.loni.usc.edu/), a multicenter publicly funded longitudinal study of individuals with AD,
$\mathrm{MCI}$, and normal cognition (NC). ADNI participants were followed longitudinally, with visits every 6 months for the first 2 years, followed by annual visits. At each follow-up visit, any change to a participant's clinical diagnosis or biomarker data was recorded in the ADNI database. Here, we restricted the present analysis to $\mathrm{MCI}$ and $\mathrm{NC}$ subjects whose biomarker data of CSF $A \beta_{42}$ (labeled " $A$ " biomarker), CSF p-tau (labeled "T" biomarker), and FDGPET (labeled "N" biomarker) were all available.

In total, 262 non-demented elderly individuals, including $159 \mathrm{MCI}$ and $103 \mathrm{NC}$, had baseline and follow-up data of ATN biomarkers identified from the ADNI cohort (Table 1 and Fig. 1).

\section{Data on CSF $A \beta$, $p$-tau, and FDG-PET}

High-quality data on CSF $A \beta_{42}$, p-tau, and FDG-PET were downloaded from the ADNI dataset. Levels of $A \beta_{42}$ and p-tau were measured from all available CSF samples as previously described [10]. Briefly, $A \beta_{42}$ and p-tau were measured using the multiplex xMAP Luminex platform (Luminex Corporation, Austin, TX) with Innogenetics (INNO-BIA AlzBio3; Ghent, Belgium) immunoassay kitbased research-use only reagents containing 4D7A3 monoclonal antibody for $\mathrm{A} \beta_{42}$, and AT270 monoclonal antibody for p-tau. All CSF biomarker assays were performed in duplicate and averaged.

The neuroimaging data of cerebral metabolic rate for glucose (CMRgl) on FDG-PET was also downloaded from the ADNI dataset. A detailed description of FDG-PET image acquisition and processing can be found at http:// adni.loni.usc.edu/data-samples/pet/. The mean FDG uptake was averaged over 5 pre-defined regions of interest (metaROIs) that are sensitive to AD-related changes in metabolism, including right and left angular gyri, right and left inferior temporal regions, and bilateral posterior cingulate. PET images were spatially normalized in statistical parametric mapping (SPM) to the MNI PET template. We extracted the mean counts from the 5 metaROIs for each subject's FDG scans at each time point, computing the intensity values with SPM subroutines. Finally, we intensity-normalized each metaROI mean by dividing it by pons/vermis reference region mean. The changes of CMRgl on FDG-PET for longitudinal analysis were observed.

Table 1 Demographic characteristics of study subjects

\begin{tabular}{llll}
\hline Characteristics & NC & MCl & Total \\
\hline Number & 103 & 159 & 262 \\
Age (mean years \pm SD) & $74.98 \pm 5.92$ & $71.75 \pm 7.46$ & $73.02 \pm 7.07$ \\
Gender (male/female) & $59 / 44$ & $91 / 68$ & $150 / 112$ \\
Education (mean years \pm SD) & $16.41 \pm 2.81$ & $16.13 \pm 2.54$ & $16.24 \pm 2.65$ \\
APOE \&4 (carrier/non-carrier) & $29 / 74$ & $78 / 81$ & $107 / 155$ \\
\hline
\end{tabular}

APOE apolipoprotein $\mathrm{E}, \mathrm{MCl}$ mild cognitive impairment, $N C$ normal cognition 


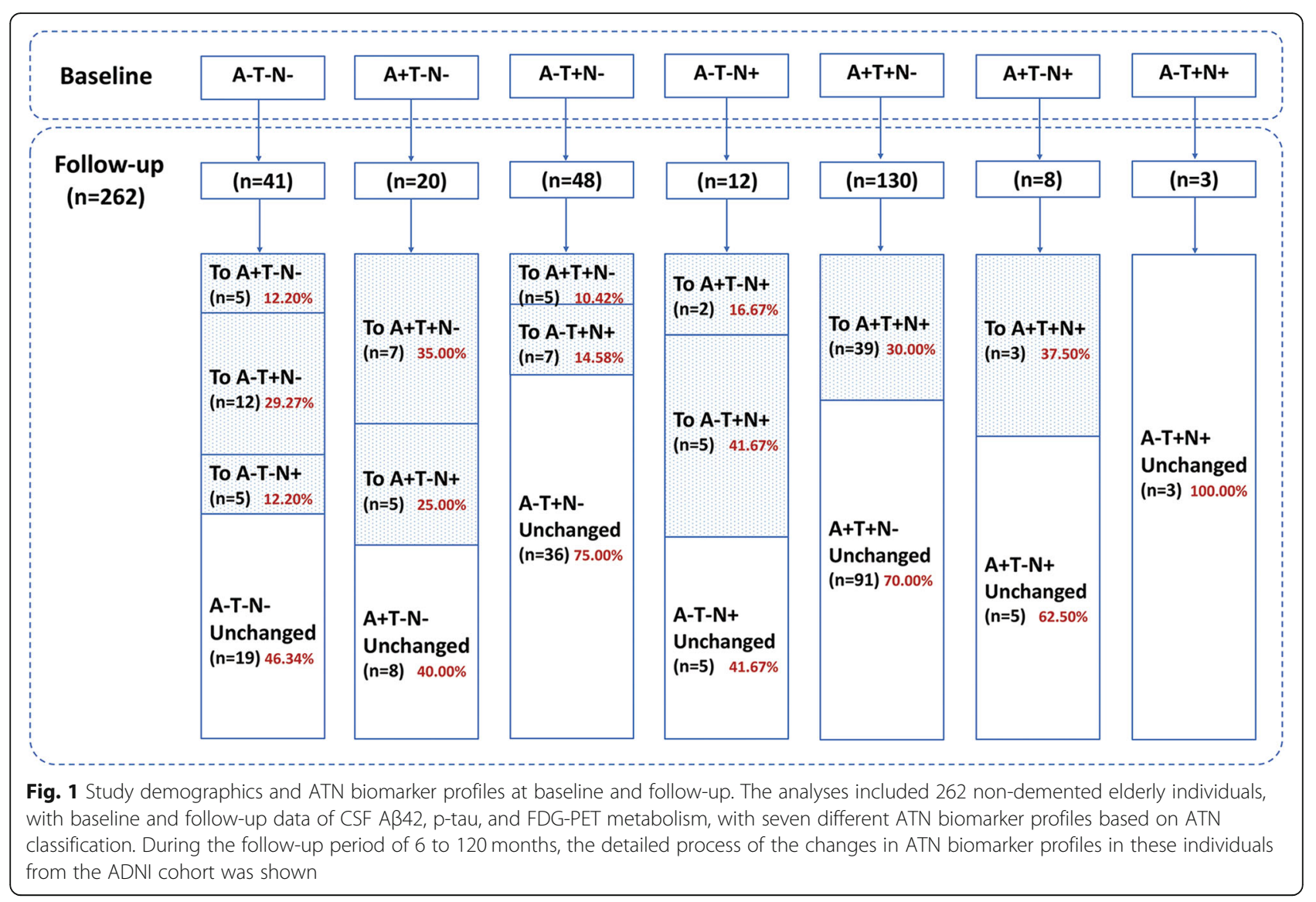

\section{ATN classification and data collection}

The cutoff values for denoting normal (negative) versus abnormal (positive) A/T/N biomarker, obtained from the extant literature $[10,11]$, might serve as signatures for the presence of $\mathrm{A} / \mathrm{T} / \mathrm{N}$ pathology. The cutoff concentrations of CSF $A \beta_{42}$ and p-tau were $192 \mathrm{pg} / \mathrm{ml}$ and $23 \mathrm{pg} / \mathrm{ml}$, respectively, while the FDG cutoff value used in this study was 1.21. Applying these cutoff values to the ATN biomarkers resulted in eight possible ATN biomarker profiles at baseline: A-T-N-, A+T-N-, A-T+N-, $\mathrm{A}-\mathrm{T}-\mathrm{N}+, \mathrm{A}+\mathrm{T}+\mathrm{N}-, \mathrm{A}+\mathrm{T}-\mathrm{N}+, \mathrm{A}-\mathrm{T}+\mathrm{N}+$, and $\mathrm{A}+\mathrm{T}+\mathrm{N}+$. Those with ATN all positive $(\mathrm{A}+\mathrm{T}+\mathrm{N}+)$ at baseline were excluded from the study. Follow-up data for the other seven ATN biomarker profiles were collected (Fig. 1). We recorded the process of the first conversion in one of the three ATN biomarkers from negative to positive during follow-up. We then analyzed the possible longitudinal patterns of biomarker profiles and estimated the conversion rate and temporal evolution of biomarker changes in different patterns throughout the course of the disease. It is worth noting that, for the present study, we did not include borderline cases $( \pm 5 \%$ from the original cutoffs for ATN biomarkers) to avoid drawing conclusions based on borderline cases. Detailed information can be found in our previously published article [12].

\section{Statistical analysis}

Demographic characteristics of our individuals are presented using means and standard deviations (SD) for continuous variables and proportions for categorical variables. To evaluate how biomarkers changed over time, we used linear mixed-effects models. The model allowed an individual's change rate of biomarker profiles to depend on his or her pathological stage by fitting a model with an interaction between time and "ATN" profiles. Subject-specific intercepts and slopes were included in random-effects models that allow for heterogeneity among subjects accounting for repeated measures on the same subject. Age, gender, educational years, and $A P O E$ $\varepsilon 4$ genotype were also included as covariates. Estimates and $95 \%$ confidence intervals (CIs) were calculated using the parametric bootstrap method in the arm package with 10,000 replicates. The baseline estimated time (months) to each biomarker change was calculated by the absolute value of the difference between the values in individuals with follow-up data, but without this biomarker change, and the cutoff values for defining positive/negative individuals of this biomarker divided by the estimated rate of change. The total conversion time for all biomarker changes in different patterns was obtained by summing the conversion time of each of the three 
ATN biomarkers. All statistical analyses were conducted using the R statistical software (www.R-project.org).

\section{Results}

The analyses included 262 non-demented elderly individuals (Table 1), with baseline and follow-up data of CSF $A \beta_{42}$, p-tau, and FDG-PET metabolism, with seven different initial ATN biomarker profiles (Fig. 1). The follow-up time ranged from 6 to 120 months, and the median follow-up time was 24 months. The number of individuals who had follow-up data over 60 months was extremely small. Figure 1 shows the detailed process of the changes in ATN biomarker profiles.

During the follow-up period of 6 to 120 months, there were four sequences in Alzheimer's ATN biomarker profiles, from all negative to all positive biomarkers through disease progression (Fig. 2), specifically A-T-N, A-N-T, T$\mathrm{A}-\mathrm{N}$, and N-A-T biomarker sequences. The overall conversion rates of these four sequences were $1.28 \%, 1.14 \%$, $0.91 \%$, and $0.76 \%$, respectively, on the basis of the current follow-up data, while $46.34 \%$ of individuals did not have any ATN biomarker changes. The most common sequence was that the A biomarker first emerged, in line with the classic amyloid cascade hypothesis.

It is noteworthy that the $\mathrm{T}$ biomarker had a higher conversion rate in the first stage (29.27\%), but the occurrence of the T-A-N biomarker sequence was only $0.91 \%$ in the process of total conversion. This is because the rate of subsequent conversion rate in A biomarker or $\mathrm{N}$ biomarker was low at $10.42 \%$ and $14.58 \%$, respectively. Meanwhile, if the $\mathrm{N}$ biomarker appeared in the second stage, then there was no A biomarker change in the final stage of conversion, that is, a T-N sequence. Similarly, if the $\mathrm{T}$ biomarker appeared in a subsequent stage of the "N-first" biomarker pathway, then the A biomarker did not change, that is, a N-T sequence.

The "A-first" biomarker pathway had two different evolutions: A-T-N and A-N-T. Although each is similar in the total conversion rate between these two patterns (1.28\% and $1.14 \%$, respectively), there was a significant difference in the total time for all the changes (67.07 and 98.14 months, respectively). Figure 3 depicts the model results of the baseline estimated months to each biomarker change, reflecting the temporal evolution of pathology over the course of the disease, and the detailed time points for each biomarker change. Interestingly, our results show that the N-A-T sequence had the fastest rate of pathological progression (mean 65.00 months), followed by the A-T-N sequence (mean 67.07 months), then the T-A-N sequence (mean 68.85 months), and finally, the A-N-T sequence (mean 98.14 months).

\section{Discussion}

AD develops following a long pre-clinical phase with abnormal CSF and imaging biomarkers [1]. The ATN biomarker system is an unbiased system for grouping biomarkers and classifying research participants by the pathologic process each measures, thus provides a flexible platform to generate or test hypotheses concerning different pathologic processes and provides prognostic information of clinical change and progression [12]. Understanding the longitudinal trajectories of Alzheimer's ATN biomarker profiles in non-demented elderly provides insight into the pathophysiological progression of $\mathrm{AD}$ and potentially stratifies the disease into subtypes according to the temporal evolution of the biomarkers.

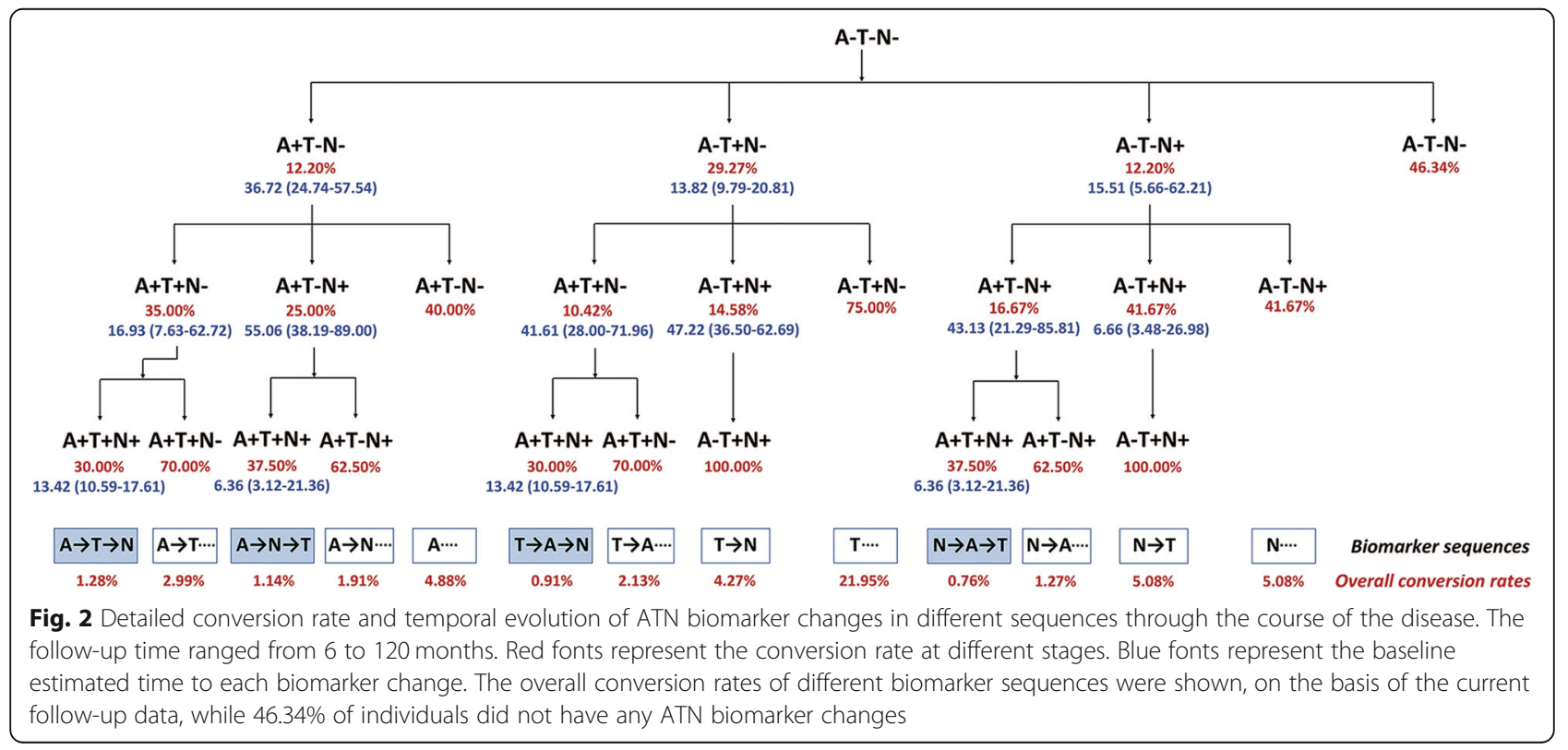




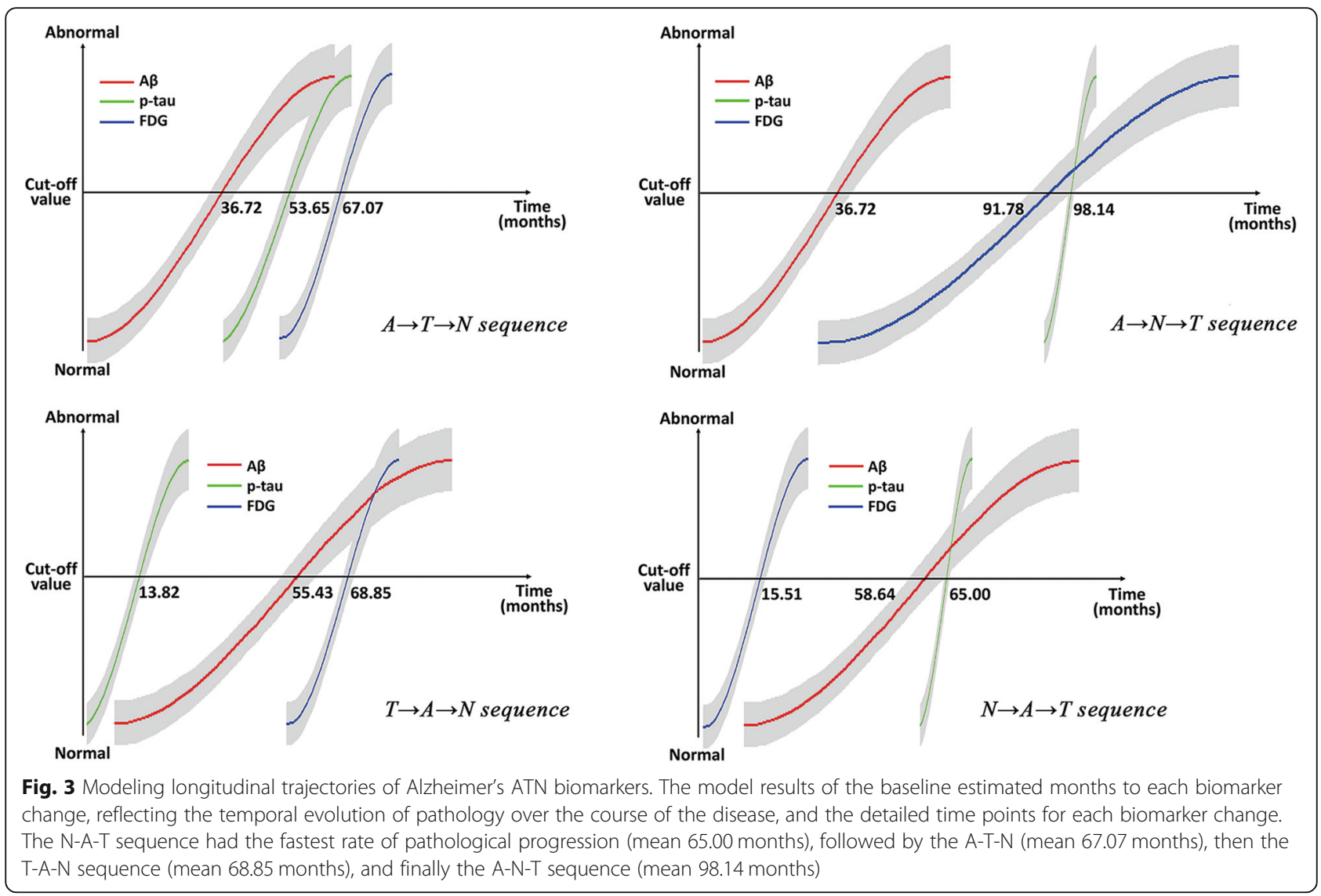

The primary goal of our current analysis was to find the possible longitudinal patterns of changes in Alzheimer's ATN biomarker profiles during the course of the disease. Our results show that there are three other longitudinal ATN biomarker pathways apart from the classical A-T-N sequence. The most common pattern is indeed that the A pathology biomarker emerges first, in line with the amyloid cascade hypothesis. The "amyloid hypothesis" has continued to gain support over the last two decades, particularly from genetic studies, and proposes that amyloid is the toxic cause of neural/synaptic damage and dementia [13]. In the current longitudinal PET study, sequential changes from $A \beta$ to tau to cognition were clearly shown [14].

Recently, abundant human and animal data implicate both $\mathrm{A}$ and $\mathrm{T}$ in the primary pathogenesis of $\mathrm{AD}[15$, 16]. The dual-pathway hypothesis suggests that $A$ and $T$ independently arise from pathophysiological processes that interact with pathogenic synergy [17]. The early stage of tauopathy, made up of the hyperphosphorylated tau, is a hallmark of several neurodegenerative disorders, and a "T-first" biomarker profile was found in our individuals from the ADNI cohort. The T-A-N sequence of pathological events previously proposed for late-onset $\mathrm{AD}$ seems to best explain the fact that the early stage of tauopathy might precede amyloid in some individuals who eventually enter the Alzheimer's continuum when they become amyloid positive $[6,18,19]$, while the T-N and $\mathrm{N}-\mathrm{T}$ sequences should be considered as nonAlzheimer's continuum profiles based on the 2018 NIAAA Research Framework [6].

In addition, many possible etiologies and proportional mixes of etiologies (for example, cell senescence or immune dysfunction) may exist for an abnormal $\mathrm{N}$ biomarker finding [20]. Although $\mathrm{N}$ biomarkers are not specific for neurodegeneration due to $\mathrm{AD}$ [6], evidence has appeared to support the idea that cognitive decline and $\mathrm{N}$ biomarker abnormalities might precede abnormal A biomarkers in some elderly individuals who later develop $\mathrm{AD}[8,21]$. Consistent with previous work, the NA-T sequence was also found in our individuals from the ADNI cohort. This pattern of longitudinal pathological changes needs to be further tested in living individuals by large sample prospective studies.

The second goal of our current analysis was to show the temporal evolution of biomarker changes through the course of the disease. Previous studies have found that CSF $A \beta_{42}$ was unequivocally abnormal 5-10 years or more prior to dementia diagnosis [22], while both CSF t-tau and $\mathrm{p}$-tau became progressively more abnormal as the time to 
diagnosis of dementia decreased [23]. Our current results are generally consistent with the above views. As can be seen in the "A-first" biomarker sequences, the A-T-N sequence had a significantly faster rate of disease progression than the A-N-T sequence. It is noteworthy that the $\mathrm{N}-\mathrm{A}-\mathrm{T}$ sequence had the fastest disease progression rate. The $\mathrm{N}$ biomarker is an indicator of neurodegeneration or neuronal injury that can result from many causes. Recent longitudinal analyses show that, even before amyloid turns officially positive, subthreshold accumulation correlates with subtle memory deficits and cortical tau deposition $[24,25]$. Therefore, we speculate that $\mathrm{A} \beta$ may be already accumulating in these $\mathrm{N}$-positive-first, but A-negative individuals, and increased $A \beta$ accumulation might be associated with faster disease progression.

However, despite the long pre-clinical disease window covered by the current study, the follow-up data especially CSF data are limited because of its invasiveness. Therefore, only small numbers of participants had longitudinal data, and the majority of longitudinal participants had conversion results of biomarker changes only once. No individual had a full conversion of biomarkers from all negative to all positive across the follow-up period, and our results represent sample rather than individual effects. In addition, we calculated the conversion rates of each biomarker sequence similarly as previous research [26], while $46.34 \%$ of individuals did not have any ATN biomarker changes based on the current follow-up data. How many individuals will undergo further changes cannot be known, because of increasing death rates with age and the difficulty in longer follow-ups. Anyway, a greater number of individuals and time points, and longer follow-up time would increase the feasibility of modeling and provide more statistically powerful results in the future.

\section{Conclusions}

To our knowledge, the current work presents a comprehensive analysis of longitudinal trajectories of Alzheimer's ATN biomarker profiles in non-demented elderly individuals from the ADNI cohort. Except for the classical A-T-N sequence, our study has demonstrated that three other longitudinal patterns of changes in Alzheimer's ATN biomarker profiles are also present, including the "A-first," "T-first," and "N-first" biomarker pathways. Understanding these longitudinal changes further demonstrates the diversity of the pathophysiological progression of the disease. Stratifying disease into subtypes depending on the temporal evolution of biomarkers could benefit the early recognition and treatment of diseases. Meanwhile, early targeted interventions blocking the effect of biomarkers might alter the natural history of the disease.

\section{Abbreviations}

$A \beta$ : Amyloid- $\beta$; AD: Alzheimer's disease; ADNI: Alzheimer's Disease Neuroimaging Initiative; ATN: Amyloid- $\beta$, pathologic Tau, or Neurodegeneration; CMRgl: Cerebral metabolic rate for glucose; CSF: Cerebrospinal fluid; FDG: Fluorodeoxyglucose; MCl: Mild cognitive impairment; NC: Normal cognition; NIA-AA: National Institute on Aging and Alzheimer's Association; PET: Positron emission tomography; $\mathrm{p}$ tau: Phosphorylated tau

\section{Acknowledgements}

Data collection and sharing was funded by ADNI. ADNI is funded by the National Institute on Aging and the National Institute of Biomedical Imaging and Bioengineering and through generous contributions from the following: Alzheimer's Association; Alzheimer's Drug Discovery Foundation; BioClinica, Inc.; Biogen Idec Inc.; Bristol-Myers Squibb Company; Eisai Inc.; Elan Pharmaceuticals, Inc.; Eli Lilly and Company; F. Hoffmann-La Roche Ltd. and its affiliated company Genentech, Inc.; GE Healthcare; Innogenetics, N.V.; IXICO Ltd.; Janssen Alzheimer Immunotherapy Research \& Development, LLC.; Johnson \& Johnson Pharmaceutical Research \& Development LLC.; Medpace, Inc.; Merck \& Co., Inc;; Meso Scale Diagnostics, LLC.; NeuroRx Research; Novartis Pharmaceuticals Corporation; Pfizer Inc.; Piramal Imaging; Servier; Synarc Inc.; and Takeda Pharmaceutical Company. The Canadian Institutes of Health Research is providing funds to support ADNI clinical sites in Canada. Private sector contributions are facilitated by the Foundation for the National Institutes of Health (www.fnih.org). The grantee organization is the Northern California Institute for Research and Education, and the study is coordinated by the Alzheimer's Disease Cooperative Study at the University of California, San Diego. ADNI data are disseminated by the Laboratory for Neuro Imaging at the University of Southern California.

Data used in the preparation of this article were obtained from the Alzheimer's Disease Neuroimaging Initiative (ADNI) database (adni.loni.usc.edu). As such, the investigators within the ADNI contributed to the design and implementation of ADNI and/or provided data but did not participate in the analysis or writing of this report. A complete listing of ADNI investigators can be found at http://adni.loni.usc.edu/wp-content/ uploads/how_to_apply/ADNI_Acknowledgement_List.pdf.

\section{Authors' contributions}

JTY conceptualized the study, analyzed and interpreted the data, and drafted and revised the manuscript. MST and XJ analyzed and interpreted the data, drafted and revised the manuscript, did the statistical analysis, and prepared all the figures. JQL, WX, HFW, and CCT analyzed and interpreted the data. $\mathrm{QD}, C T Z$, LT, and JS interpreted the data and revised the manuscript. All authors contributed to the writing and revisions of the paper and approved the final version. Data used in the preparation of this article were obtained from the Alzheimer's Disease Neuroimaging Initiative (ADNI) database (adni. loni.usc.edu). As such, the investigators within the ADNI contributed to the design and implementation of ADNI and/or provided data but did not participate in the analysis or writing of this report.

\section{Funding}

This study was supported by grants from the National Natural Science Foundation of China $(91849126,81571245,81501103)$, the National Key R\&D Program of China (2018YFC1314700), Taishan Scholars Program of Shandong Province (tsqn20161078), Shanghai Municipal Science and Technology Major Project (No.2018SHZDZX01) and Zhangjiang Lab, Tianqiao and Chrissy Chen Institute, and the State Key Laboratory of Neurobiology and Frontiers Center for Brain Science of Ministry of Education, Fudan University.

\section{Availability of data and materials}

The dataset generated and analyzed in the current study is available from the corresponding author on reasonable request.

\section{Ethics approval and consent to participate}

The study was approved by the institutional review boards of all participating institutions, and written informed consent was obtained from all participants or their guardians according to the Declaration of Helsinki (consent for research). 


\section{Competing interests}

The authors declared no potential conflicts of interest with respect to the research, authorship, and/or publication of this article.

\section{Author details}

'Department of Neurology, Qingdao Municipal Hospital, Qingdao University, Qingdao, China. ${ }^{2}$ Department of Neurology, Dalian Medical University, Dalian, China. ${ }^{3}$ Department of Neurology and Institute of Neurology, Huashan Hospital, Shanghai Medical College, Fudan University, 12th Wulumuqi Zhong Road, Shanghai 200040, China. ${ }^{4}$ PET Center, Huashan Hospital, Fudan University, Shanghai, China. ${ }^{5}$ Department of Psychiatry, University of Cambridge, Cambridge, UK. ${ }^{6}$ Medical Research Council and Wellcome Trust Behavioural and Clinical Neuroscience Institute, University of Cambridge, Cambridge, UK. ${ }^{7}$ Cambridgeshire and Peterborough NHS Trust, Cambridge, UK.

Received: 21 October 2019 Accepted: 24 April 2020

Published online: 11 May 2020

\section{References}

1. Sperling RA, Aisen PS, Beckett LA, Bennett DA, Craft S, Fagan AM, et al. Toward defining the preclinical stages of Alzheimer's disease: recommendations from the National Institute on Aging-Alzheimer's Association workgroups on diagnostic guidelines for Alzheimer's disease. Alzheimers Dement. 2011;7:280-92.

2. Elias MF, Beiser A, Wolf PA, Au R, White RF, D'Agostino RB. The preclinical phase of Alzheimer disease: a 22-year prospective study of the Framingham Cohort. Arch Neurol. 2000;57:808-13.

3. Pike KE, Savage G, Villemagne VL, Ng S, Moss SA, Maruff P, et al. Betaamyloid imaging and memory in non-demented individuals: evidence for preclinical Alzheimer's disease. Brain. 2007;130:2837-44.

4. McKhann GM, Knopman DS, Chertkow H, Hyman BT, Jack CR Jr, Kawas CH, et al. The diagnosis of dementia due to Alzheimer's disease: recommendations from the National Institute on Aging-Alzheimer's Association workgroups on diagnostic guidelines for Alzheimer's disease. Alzheimers Dement. 2011;7:263-9.

5. Albert MS, DeKosky ST, Dickson D, Dubois B, Feldman HH, Fox NC, et al. The diagnosis of mild cognitive impairment due to Alzheimer's disease: recommendations from the National Institute on Aging-Alzheimer's Association workgroups on diagnostic guidelines for Alzheimer's disease. Alzheimers Dement. 2011;7:270-9.

6. Jack CR Jr, Bennett DA, Blennow K, Carrillo MC, Dunn B, Haeberlein SB, et al. NIA-AA Research Framework: toward a biological definition of Alzheimer's disease. Alzheimers Dement. 2018;14:535-62.

7. Jack CR Jr, Knopman DS, Jagust WJ, Petersen RC, Weiner MW, Aisen PS, et al. Tracking pathophysiological processes in Alzheimer's disease: an updated hypothetical model of dynamic biomarkers. Lancet Neurol. 2013; 12:207-16.

8. Jack CR Jr, Holtzman DM. Biomarker modeling of Alzheimer's disease. Neuron. 2013;80:1347-58.

9. Landau SM, Frosch MP. Tracking the earliest pathologic changes in Alzheimer disease. Neurology. 2014;82:1576-7.

10. Shaw LM, Vanderstichele H, Knapik-Czajka M, Clark CM, Aisen PS, Petersen RC, et al. Cerebrospinal fluid biomarker signature in Alzheimer's disease neuroimaging initiative subjects. Ann Neurol. 2009;65:403-13.

11. Landau SM, Harvey D, Madison CM, Reiman EM, Foster NL, Aisen PS, et al. Comparing predictors of conversion and decline in mild cognitive impairment. Neurology. 2010;75:230-8.

12. Yu JT, Li JQ, Suckling J, Feng L, Pan A, Wang YJ, et al. Frequency and longitudinal clinical outcomes of Alzheimer's AT(N) biomarker profiles: a longitudinal study. Alzheimers Dement. 2019;15:1208-17.

13. Hardy JA, Higgins GA. Alzheimer's disease: the amyloid cascade hypothesis. Science. 1992;256:184-5.

14. Hanseeuw BJ, Betensky RA, Jacobs HIL, Schultz AP, Sepulcre J, Becker JA, et al. Association of amyloid and tau with cognition in preclinical Alzheimer disease: a longitudinal study. JAMA Neurol. 2019;76(8):915-24.

15. Bateman RJ, Xiong C, Benzinger TL, Fagan AM, Goate A, Fox NC, et al. Clinical and biomarker changes in dominantly inherited Alzheimer's disease. N Engl J Med. 2012;367:795-804.
16. Chabrier MA, Blurton-Jones M, Agazaryan AA, Nerhus JL, Martinez-Coria $\mathrm{H}_{\text {, }}$ LaFerla FM. Soluble Aß promotes wild-type tau pathology in vivo. J Neurosci. 2012;32:17345-50.

17. Bloom GS. Amyloid-beta and tau: the trigger and bullet in Alzheimer disease pathogenesis. JAMA Neurol. 2014;71:505-8.

18. Braak H, Braak E. Frequency of stages of Alzheimer-related lesions in different age categories. Neurobiol Aging. 1997;18:351-7.

19. Price JL, Morris JC. Tangles and plaques in nondemented aging and "preclinical" Alzheimer's disease. Ann Neurol. 1999;45:358-68.

20. Wyss-Coray T. Ageing, neurodegeneration and brain rejuvenation. Nature. 2016:539:180-6

21. Drachman DA. The amyloid hypothesis, time to move on: amyloid is the downstream result, not cause, of Alzheimer's disease. Alzheimers Dement. 2014;10:372-80

22. Buchhave $\mathrm{P}$, Minthon L, Zetterberg $\mathrm{H}$, Wallin AK, Blennow $\mathrm{K}$, Hansson $\mathrm{O}$. Cerebrospinal fluid levels of beta-amyloid 1-42, but not of tau, are fully changed already 5 to 10 years before the onset of Alzheimer dementia. Arch Gen Psychiatry. 2012;69:98-106.

23. Fagan AM, Mintun MA, Shah AR, Aldea P, Roe CM, Mach RH, et al. Cerebrospinal fluid tau and ptau(181) increase with cortical amyloid deposition in cognitively normal individuals: implications for future clinical trials of Alzheimer's disease. EMBO Mol Med. 2009:1:371-80.

24. Landau SM, Horng A, Jagust WJ. Memory decline accompanies subthreshold amyloid accumulation. Neurology. 2018;90:e1452-e60.

25. Leal SL, Lockhart SN, Maass A, Bell RK, Jagust WJ. Subthreshold amyloid predicts tau deposition in aging. J Neurosci. 2018;38:4482-9.

26. Jack CR Jr, Therneau TM, Wiste HJ, Weigand SD, Knopman DS, Lowe VJ, et al. Transition rates between amyloid and neurodegeneration biomarker states and to dementia: a population-based, longitudinal cohort study. Lancet Neurol. 2016;15:56-64.

\section{Publisher's Note}

Springer Nature remains neutral with regard to jurisdictional claims in published maps and institutional affiliations.

Ready to submit your research? Choose BMC and benefit from:

- fast, convenient online submission

- thorough peer review by experienced researchers in your field

- rapid publication on acceptance

- support for research data, including large and complex data types

- gold Open Access which fosters wider collaboration and increased citations

- maximum visibility for your research: over $100 \mathrm{M}$ website views per year

At BMC, research is always in progress.

Learn more biomedcentral.com/submissions 